\title{
Tendencias de consumo de queso mozzarella en la ciudad de Quito
}

Fecha de recepción: 23/10/2019 • Fecha de aceptación: 06/12/2019 • Fecha de publicación:10/02/2020

Georgina Retamal

Edesa Ecuador

paoretcor@yahoo.com

https://orcid.org/0000-0003-2892-3272

Eduardo Pastas

Universidad Israel

rpastas@uisrael.edu.ec

https://orcid.org/0000-0002-0769-3888

Miguel Aizaga

Universidad Israel

maizaga@uisrael.edu.ec

https://orcid.org/0000-0002-4492-1181

\section{RESUMEN:}

La presente investigación explica el plan de negocios para la creación de una empresa distribuidora de queso mozzarella, ubicada en el Distrito Metropolitano de Quito, con el fin de satisfaga las necesidades de los clientes y obtener ganancias para sus inversionistas. El marco teórico detalla los conceptos teóricos importantes para sustentar la investigación; se han citado trabajos de investigación previos, cuyos temas están relacionados con el problema de presente trabajo. Se utilizó el método cuantitativo, estudio de campo; $y$, como herramienta de recolección de datos se diseñó una encuesta para conocer las preferencias del mercado, se realizó el cálculo de la población y muestra, para finalmente describir los resultados obtenidos, los cuales fueron favorables para la puesta en marcha del proyecto. Debido a que el estudio de mercado reveló la existencia de una demanda insatisfecha en el sector donde se pretende ejecutar el proyecto, se diseñó el proceso de organización, gestión empresarial, de servicio y ambiental aplicables al presente proyecto, del mismo modo, se identifica- 
ron los requisitos legales para la puesta en marcha del proyecto, finalmente se realizó el cálculo del estudio financiero, donde se obtuvo una TMAR de 13,71\%, un VAN de $\$ 91.711,87$, una TIR de 83,13 $\%$, se calculó que el período para la recuperación de la inversión inicial es de 4 años, 0 meses y 6 días de la puesta en marcha del proyecto, estos resultados son ya que indican que el plan es viable.

PALABRAS CLAVE: Demanda Insatisfecha, Microempresas, Agentes Intermediarios, Canasta Básica Familiar.

ABSTRACT:

This research explains the business plan for the creation of a mozzarella cheese distribution company, located in the Quito Metropolitan District, in order to meet the needs of customers and obtain profits for its investors. The theoretical framework details the important theoretical concepts to support the research; previous research has been cited, the topics of which are related to the problem of the present work. The quantitative method was used, field study; and, as a data collection tool, a survey was designed to know the preferences of the market, the population and sample were calculated, to finally describe the results obtained, which were favorable for the implementation of the project. Since the market study revealed the existence of unsatisfied demand in the sector where the project is to be implemented, the organization, business management, service and environmental processes applicable to the present project were designed. Similarly, the legal requirements for the implementation of the project were identified, and finally the financial study was calculated, obtaining a TMAR of $13.71 \%$, a NPV of $\$ 91.711 .87$, an IRR of $83.13 \%$, it was calculated that the period for the recovery of the initial investment is 4 years, 0 months and 6 days from the start of the project, these results are since they indicate that the plan is viable.

KEYWORDS: Unsatisfied Demand, Micropresses, Intermediate Agents, Basic Family Basket 


\section{INTRODUCCIÓN}

El consumo de leche en el mundo se remonta a diez siglos antes de Cristo, cuando el ser humano dejaba la vida nómada y comenzó a domesticar animales para proveerse de alimentos, manteniendo crías de estos animales para reservar la leche sobrante para su alimentación. Con el paso del tiempo surge la necesidad de abastecer a la población de leche, por lo cual se busca desarrollar procedimientos para extender la vida útil de éste producto, mediante la elaboración del queso, finalmente, desde el descubrimiento de la pasteurización y posteriormente de procesos térmicos, se ha ido favoreciendo el desarrollo de la industrialización de leche y sus derivados, así como también desarrollo local, productivo, oportunidades laborales, etc. (Centro de la Industria Láctea del Ecuador, 2015)

En el país post - colombino, durante el año 1544 se expide una ordenanza para venta de queso por libras, para lo cual, se fija un precio, según el autor W. Stevenson, citado por el Centro de Industria Láctea del Ecuador (CIL) este producto era muy consumido en Quito, a pesar de que la historia no especifica el tipo de queso que en ese tiempo se comercializaba (Centro de la Industria Láctea del Ecuador, 2015)

Actualmente el queso constituye un alimento consumido ampliamente a nivel mundial y sus características sensoriales, nutritivas, funcionales, etc., difieren dependiendo del tipo de queso (maduro, semi - maduro, fresco), inclusive su forma de preparación ha sufrido cambios debido a la transformación y desarrollo tecnológico e industrial (Ramórez López \& Vélez Ruiz , 2012)

En referencia a los que dice el CIL (2018), el sector lechero en el Ecuador ha mejorado su producción y ha crecido de manera rentable, esto se refleja en las cifras presentadas el año pasado, ya que la producción de leche ascendió a 5,3 millones de litros por día (Centro de la Industria Láctea del Ecuador, 2018)

Conforme dice Orozco (2015), los ecuatorianos demandan cada vez más queso, de la producción total de leche al día, más de un tercio se destina a la elaboración de este producto, lo que hace que la industria quesera crezca cada año.

Como se puede observar, el mercado presenta cifras atractivas para la apertura de nuevos emprendimientos dedicados a la elaboración y comercialización de quesos, razón por la cual, se ha visto la necesidad de la creación de una empresa distribuidora de queso mozzarella ubicada en el Distrito Metropolitano de Quito, para satisfacer las necesidades de consumo creciente de este delicioso producto a los hogares de esta ciudad.

El consumo de leche y sus derivados sigue siendo bajo en Ecuador, lo que hace que el sector público y privado ponga en marcha campañas para incentivar el consumo de estos productos dentro del país (El Telégrafo, 2019), por esta razón, la creación de empresas dedicadas no solo a la producción sino también a la distribución de estos productos, es beneficioso, no solamente para la economía del país sino como un aporte a la buena alimentación de la población. 
Partiendo de ésta problemática, se busca impulsar el consumo de queso mozzarella, tomando en cuenta la necesidad de proveer un producto de consumo masivo de calidad y a bajos costos en el mercado quiteño, donde se ha detectado una oportunidad para la comercialización de queso mozzarella en un nicho que no ha sido completamente atendido y que requiere ser provisionado por una empresa legalmente conformada, que brinde una imagen confiable a sus clientes, que comercialice este delicioso producto, y realice las entregas a domicilio, es decir, ofrezca un beneficio extra al cliente.

La presente investigación pretende enriquecer el conocimiento, abriendo una alternativa que permita fomentar el trabajo artesanal de elaboración de queso mozzarella, así como a los inversionistas, proveer información útil sobre la factibilidad de la puesta en marcha del proyecto.

\section{METODOLOGÍA Y MATERIALES}

\subsection{Contextualización espacio temporal del problema}

\subsubsection{Macro entorno}

En referencia a Tapia (2017), a pesar de que Ecuador es uno de los países más pequeños, el índice de emprendimiento ha ido aumentando significativamente cada año, posicionando al país con el porcentaje más alto en comparación a otros países de la región.

De acuerdo con la publicación de El Universo (2018), el Ecuador se posicionó como líder en la región, por sexto año consecutivo en el índice de Actividad Emprendedora Temprana (TEA), según lo indican los resultados de la medición realizada por la Escuela de Negocios de la ESPOL, en la cual se mide el emprendimiento en 66 países (equivalente al 69,2\% de la población mundial).

El mencionado estudio muestra, además, que en 2017 , cerca de 3 millones de adultos (29.6\% de la población entre 18 y 64 años), pusieron en marcha un emprendimiento o poseían un negocio con menos de 42 meses de antigüedad, correspondiente a la TEA más alta de la región, seguido por Perú y Chile (El Universo, 2018)

De acuerdo con un reporte del INEC, publicado en 2016, la población en el Ecuador en el período de 2002 al 2012, tuvo una tasa de crecimiento del 2\%, en la provincia del Pichincha del 2,1\% y en cantón Quito 1,4\% promedio anual, mientras que, a juzgar por el Banco Mundial (2017), la tasa de crecimiento demográfico del Ecuador se estimó en 1,5\%, lo que proporciona una perspectiva favorable del mercado potencial.

Por otra parte, Más Leche - CIL (2017) menciona que, la ganadería ha sido tradicionalmente un sector clave para la economía nacional, razón por la cual, se ha puesto especial interés en planes de mejora para los procesos dentro de la industria láctea.

De acuerdo al Banco Central del Ecuador en la información emitida sobre Producto Interno Bruto (2015), se indica, que este sector lácteo aporta alrededor del 7,59\% del PIB del país, por ello, se 
prioriza, tomar acciones y prácticas responsables para que siga siendo una industria sostenible.

\subsubsection{Meso entorno}

De acuerdo con lo señalado por la Unidad de Estadísticas Agropecuarias (2017), la producción lechera se concentra mayormente en la región sierra con un 64,31\% de la producción nacional de leche, seguido por la región Costa con el 29,99\% y finalmente el Oriente con el 5,67\%, demás, el uso y destino de la producción lechera en el país tiene un comportamiento regular.

Conforme dice el INEC (2014), la provincia que mayor aporte presenta en la producción de leche es Pichincha, con una participación del 13.94\% del total de la producción del país, se puede agregar que según Lácteos LATAM (2017), de la producción para consumo humano, el 11,5\% se destina a queso mozzarella, el 35\% a queso artesanal, el 6\% para derivados lácteos como yogurt, queso maduro, el restante se destina a consumo directo y comercialización.

La Dirección Nacional de Estudios de Mercado indica que, "actualmente, el sector pecuario representa el 3\% del Producto Interno Bruto, mientras que el sector lácteo representa un 1,4\% aproximadamente. De acuerdo a la información obtenida del INEC, el sector genera empleo directo al 9\% de la población económicamente activa" (2019, pág. 18)

\subsubsection{Micro entorno}

De acuerdo con la Dirección Nacional de Estudios de Mercado (2019), existen 65 operadores económicos que se encuentran registrados en la Superintendencia de Compañías y Seguros bajo la actividad económica de pasteurizadoras de leche, sin embargo, solo cuatro operadoras tienen capacidad de procesamiento de leche mayor a 250.000 litros diarios, entre ellas, Pasteurizadora Quito S.A., cuya planta opera en el Distrito Metropolitano de Quito.

Ulcuango Conlago (2017), menciona que actualmente se comercializan quesos de diferentes precios y tamaños en Quito y la competencia es notoria debido a la presencia de grandes y reconocidas marcas como Toni, Salinerito, Kiosco, entre otros, las cuales, cuentan además con canales de distribución establecidos, puntos de venta a nivel local, nacional e incluso, internacional.

\subsection{Cuerpo teórico conceptual}

\subsubsection{Plan de negocio}

"Documento que delinea el concepto esencial sobre el que una empresa se fundamentará y que describe la forma en que se materializará dicho concepto" (Longenecker, Palich, Petty, \& Hoy, 2012), según los mismos autores, en el plan de negocios debe presentarse cuál es la idea básica que se pretende desarrollar, así como responderse interrogantes sobre dónde se encuentra la idea de emprendimiento, hacia dónde se desea llegar y cómo se lo pretende poner en marcha. 


\section{Objetivos del plan de negocios}

De acuerdo con Longenecker, Palich, Petty, y Hoy (2012), el plan de negocios debe perseguir los siguientes objetivos fundamentales:

- Identificar la naturaleza y contexto de la oportunidad de negocios: en este apartado se debe explicar la razón de la existencia de la oportunidad.

- Desarrollar el enfoque que se plantea utilizar para aprovechar la oportunidad que se ha identificado.

- Identificar los factores que demostrarán el éxito o fracaso de la iniciativa de emprendimiento.

De acuerdo a Longenecker, Palich, Petty, y Hoy (2012), el plan de negocios, en resumidas cuentas, "es una herramienta para exponer las metas y estrategias que los agentes internos de la empresa utilizarán, y para ayudar a desarrollar las relaciones con personas externas (inversionistas y otras) que podrían ayudar a la empresa a alcanzar sus metas" (Longenecker, Palich, Petty, \& Hoy, 2012, págs. 66-67)

\section{Estructura del plan de negocios}

Romero Hidalgo, Hidalgo Sánchez, y Correa Guaicha (2018), indican que el diseño de un plan de negocios se conforma por:

- Desarrollo de la idea: identificación de la idea de negocios y descripción de cómo se puede desarrollar el emprendimiento.

- Análisis de mercado: determinar cómo se puede desarrollar el plan de negocios mediante el análisis del mercado, identificado un mercado potencial para satisfacer una necesidad específica.

- Análisis técnico: análisis técnico para la gestión de la capacidad instalada de la empresa, se describe el producto o servicio y los procesos a seguir para ser comercializado en el mercado.

- Análisis económico: en este apartado se analiza la situación económica de la empresa se realizan proyecciones que permitan tener una idea de cómo evolucionarán los resultados de la puesta en marcha del negocio.

- Análisis financiero: se identifica cómo se gestionarán los gastos y la recuperación de la inversión mediante las actividades del negocio.

- Análisis ambiental: se manejan políticas de responsabilidad social y ambiental que buscan disminuir el impacto medio ambiental donde se pretende desarrollar la actividad. 


\subsubsection{Investigación de mercados}

\subsubsection{La empresa}

"La empresa es la unidad económica de producción encargada de combinar los factores o recursos productivos, trabajo, capital y recursos naturales, para producir bienes y servicios que después se venden en el mercado." (Bustamante, 2009, pág. 4).

\section{Beneficios en la empresa}

Bustamante (2009), menciona que, los beneficios para la empresa es la diferencia existente entre los ingresos y los costos.

"Los ingresos son las cantidades que obtiene la empresa por la venta de sus bienes o servicios durante un periodo determinado. Los costes son los gastos ligados a la producción de los bienes o servicios vendidos durante el periodo considerado" (Bustamante, 2009, pág. 6)

\subsubsection{Demanda}

OkDiario(2018) menciona que, la demanda se puede definir como "la cantidad de servicio o bien que una persona o un grupo de ellas desean adquirir".

\subsubsection{Demanda insatisfecha}

Referente a la demanda insatisfecha, "Descubrir una necesidad o demanda latente, presenta la oportunidad de introducir un nuevo producto social. La tarea de los expertos, es transformar la demanda latente en demanda real por medio de un producto o servicio eficaz" (WordPress, 2013).

\subsubsection{Oferta}

De acuerdo con OkDiario(2018), la oferta "sería un servicio o un bien que un vendedor pone a la venta. Dicho bien puede ser cualquier cosa, desde comida hasta servicios como clases de matemáticas o acompañamiento a personas mayores, por hablar de ejemplos sencillos y fácilmente comprensibles" (OkDiario, 2018)

\subsubsection{Los quesos}

En referencia a la Organización de las Naciones Unidas para la Alimentación y la Agricultura:

Los quesos se obtienen mediante la coagulación de la proteína de la leche (caseína), que se separa del suero [...] Los quesos pueden ser duros, semiduros, blandos madurados o no madurados. Las distintas características de los quesos derivan de las diferencias en la composición de la leche y los tipos de esta, los procedimientos de elaboración aplicados y los 
microorganismos utilizados (Organización de las Naciones Unidas para la Alimentación y la Agricultura, s.f.),

\subsubsection{La caseína}

Es la principal proteína de la leche y se utiliza como ingrediente en varios productos, entre estos: quesos, productos de pastelería, pinturas y colas. Se obtiene de la leche desnatada mediante precipitación con el cuajo o mediante bacterias inocuas productoras de ácido láctico (Organización de las Naciones Unidas para la Alimentación y la Agricultura, s.f.)

\subsubsection{El queso Mozzarella}

De acuerdo con el portal web soloquesos.com (s.f.), el queso mozzarella tiene su origen en Italia, en pocos países como Italia y Bulgaria este tipo de quesos se realizan con leche de búfalos de agua, razón por la cual, la mayoría de los quesos en el resto del mundo se elaboran con leche de vaca. Este queso no se añeja como la mayoría de los quesos, se come fresco y preferentemente en pocas horas después de su elaboración.

\subsubsection{Tipos de queso Mozarrella}

El portal web soloquesos.com (s.f.) indica que, existen varios tipos de queso mozzarella, tales como:

- "La mozzarella di bufala": quero elaborado con leche de búfalo de agua.

- "La mozzarella fior di latte": producida con leche de vaca fresca, pasteurizada o no pasteurizada.

- "La mozzarella affumicata": queso mozzarella ahumado.

- "La mozzarella baja en humedad": hecha con leche desnatada, es utilizada usualmente en la industria de servicios alimenticios.

\subsubsection{Propiedades del queso mozzarella y beneficios para la salud}

Según OKDIARIO (2017), el queso mozzarella contiene riboflavina, tianina, vacina, biotina y vitaminas A, B6, D y E; una porción de queso contiene 183mg de calcio, aporta fósforo, potasio, hierro, sodio y zinc, además de constituir una buena fuente de proteína.

El mismo autor menciona los siguientes beneficios:

- Al constituir una fuente de proteínas, es importante para recuperar energías y mantener los músculos en forma.

- Ayuda a combatir la fatiga muscular y favorece el correcto funcionamiento del cerebro. 
- Protege contra el cáncer de mama, enfermedades cardiovasculares y el síndrome metabólico.

- Ayuda a mantener una piel sana.

- Favorece la formación de glóbulos rojos.

- Debido a su contenido de vitaminas A, D y E, ayuda a la formación de los huesos, facilitan la absorción de calcio y protege las membranas celulares.

- Su contenido en biotina es favorable para las mujeres embarazadas, fortalece las uñas y regula los niveles de glucosa en sangre.

- Previene la anemia, ayuda a controlar el colesterol y previene la aparición de diabetes y artritis.

- Facilita la digestión y el buen funcionamiento de los riñones.

- Reduce la presión arterial y ayuda a rectificar los ritmos cardíacos descompensados.

\section{RESULTADOS}

\subsection{Enfoque metodológico}

"El enfoque de investigación es un proceso sistemático, disciplinado y controlado y directamente relacionada a los métodos de investigación" (Espinoza Montes, 2015, pág. 21)

\subsubsection{Método cuantitativo}

En referencia a Espinoza Montes (2015), la investigación con enfoque cualitativo “...utiliza la recolección y el análisis de datos para contestar preguntas de investigación y probar hipótesis establecidas previamente, y confiar en la medición numérica, el conteo, y el uso de la estadística para intentar establecer con exactitud patrones en una población." (pág. 21). El presente estudio investigativo se considera cuantitativo debido a que se necesitará realizar cálculos estadísticos para su posterior análisis y conclusión, esto permitirá aportar en la investigación en conocimiento de las características de la oferta y demanda, para establecer la existencia o no de una demanda insatisfecha.

\subsubsection{Estudio de campo}

Espinoza Montes (2015) menciona que, la investigación de campo requiere de un conjunto de técnicas e instrumentos para recopilar información, entre estas herramientas se encuentra la entrevista, la encuesta, el cuestionario y los talleres con grupo focales. El presente trabajo de investigación considera el estudio de campo, debido a la importancia en la aplicación de distintas herramientas de recolección de información que permitan conocer las características del mercado, sus gustos, preferencias y hábitos de consumo. 


\subsubsection{Encuesta}

"Se realiza cuando la información que se necesita puede ser obtenida a partir de la respuesta que una o varias personas puedan dar a un cuestionario preelaborado, y las mismas están dispuestas a colaborar con la investigación”. (Hernández León \& Coello Gozález, 2006, pág. 68). En la presente investigación se aplicará una encuesta a las personas que viven en el sector donde se plantea la puesta en marcha del proyecto (Anexo I).

\subsection{Población y Muestra}

\subsubsection{Población}

Para realizar el cálculo del tamaño de la población, se ha elaborado el cuadro con las variables de segmentación del mercado, especificadas para el presente proyecto:

Tabla 1

Segmentación del proyecto

\begin{tabular}{ll}
\hline Variables de Segmentación & Perfil del Cliente \\
\hline GEOGRÁFICA & \\
\hline País & Ecuador \\
\hline Provincia & Pichincha \\
\hline Cantón & Quito \\
\hline Zona & Urbana \\
\hline Administración Zonal & Eugenio Espejo \\
\hline DEMOGRÁFICA & \\
\hline Genero & Masculino, Femenino \\
\hline Edad & Entre 18 a 54 años \\
\hline PSICOGRÁFICA & \\
\hline Estilo de Vida & Personas que les guste consumir \\
\hline
\end{tabular}

Nota: elaboración propia.

Para realizar el cálculo descrito a continuación, se ha tomado información publicada por el INEC en el año 2018: 
Tabla 2

Población económicamente activa en edades de 18 a 54 años que pertenecen a la Administración Zonal Eugenio Espejo

\begin{tabular}{lr}
\hline Personas que viven en el D1 istrito Metropolitano de Quito & 1 '978.376 \\
\hline \% de personas que pertenecen a la Administración Zonal Eugenio \\
Espejo \\
\hline $\begin{array}{l}\text { Total personas que forman parte de la Administración Zonal } \\
\text { Eugenio Espejo }\end{array}$ \\
\hline$\%$ de PEA & $\mathbf{3 5 9 . 2 7 3}$ \\
\hline Total población económicamente activa & $\mathbf{2 3 4 . 2 4 6}$ \\
\hline$\%$ Personas en edades comprendidas entre 18 a 54 años & $46.55 \%$ \\
\hline $\begin{array}{l}\text { Total población económicamente activa en edades } \\
\text { comprendidas entre 18 y } \mathbf{5 4} \text { años que pertenecen a la } \\
\text { Administración Zonal Eugenio Espejo }\end{array}$
\end{tabular}

Nota: elaboración propia: Fuente: INEC.

\subsubsection{Tamaño de la muestra}

Hernández León \& Coello Gozález (2006) menciona que la muestra "es un grupo relativamente características de la población. pequeño de unidades de estudio que representa las características de la población" (pág. 51)

Para calcular la muestra utilizaremos la siguiente fórmula:

$\mathrm{n}=$ El tamaño de la muestra.

$Z=$ Valor obtenido mediante niveles de confianza 95\% de confianza equivale a 1,96

$\mathrm{N}=$ Tamaño de la población es de 109.042

$E=$ Límite aceptable de error muestral 5\% $(0,05)$.

$p=50 \%$ de éxito $(0,50)$

$q=(1-p) 50 \%$ de fracaso $(0,50)$

$$
n=383
$$




\subsection{Formas de procesamiento de la información obtenida de la aplicación de los métodos técnicos}

\subsubsection{Resultado de la Encuesta}

Una vez recolectados los datos mediante la aplicación de encuestas, se procede a realizar el análisis respectivo, mediante el uso de la herramienta Excel.

(Anexo 1)

Genero

Tabla 3

Género

\begin{tabular}{lll}
\hline Opciones & $\mathrm{Q}$ & $\%$ \\
\hline Femenino & 233 & $61 \%$ \\
\hline Masculino & 150 & $39 \%$ \\
\hline Total personas & $\mathbf{3 8 3}$ & $\mathbf{1 0 0} \%$ \\
\hline
\end{tabular}

Nota: elaboración propia.

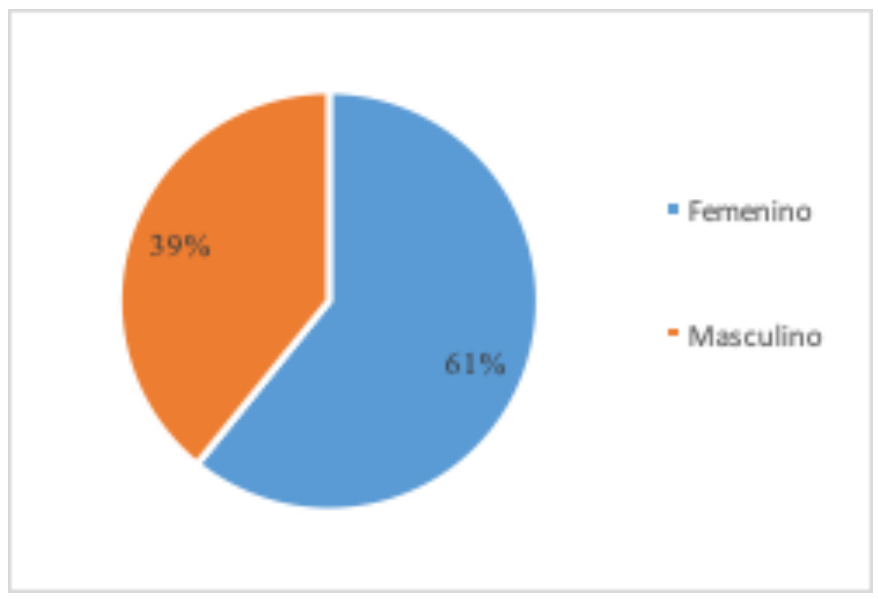

Figura 1. Género

Interpretación:

Los encuestados fueron conformados en un $61 \%$ por mujeres y $39 \%$ por hombres. 


\section{Edad:}

Tabla 4

Edad

\begin{tabular}{lll}
\hline Opciones & $\mathrm{Q}$ & $\%$ \\
\hline De 18 a 25 años & 33 & $9 \%$ \\
\hline De 26 a 33 años & 73 & $19 \%$ \\
\hline De 34 a 41 años & 153 & $40 \%$ \\
\hline De 42 a 49 años & 77 & $20 \%$ \\
\hline De 50 a 54 años & 18 & $5 \%$ \\
\hline Más de 54 años & 29 & $8 \%$ \\
\hline Total personas & $\mathbf{3 8 3}$ & $\mathbf{1 0 0} \%$ \\
\hline
\end{tabular}

Nota: elaboración propia.

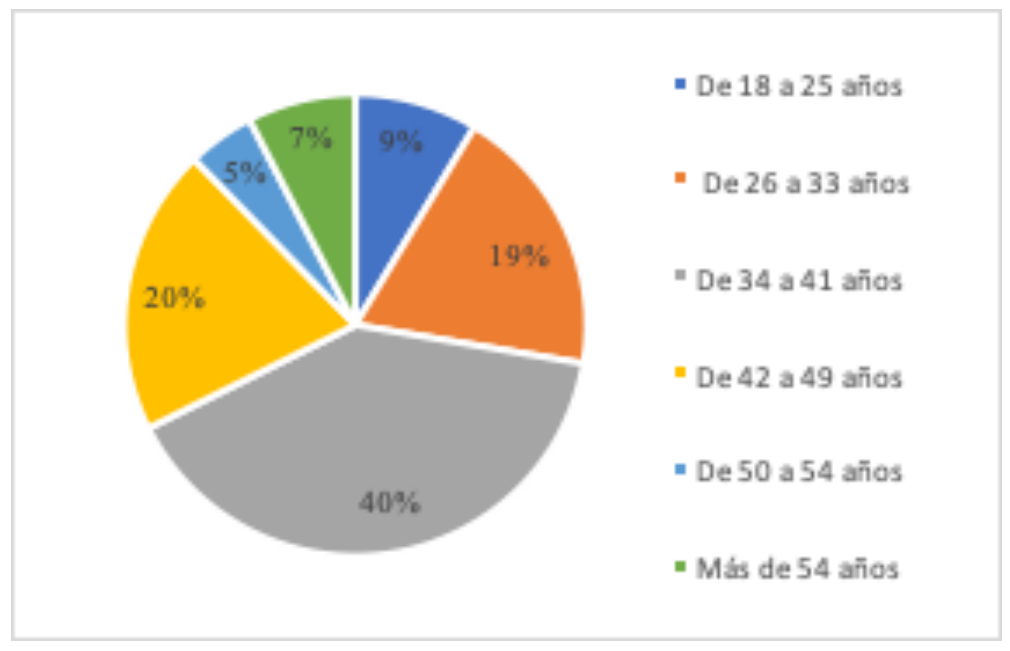

Figura 2. Edad

\section{Interpretación:}

El 9\% de encuestados tienen edades comprendidas entre 18 a 25 años, el $19 \%$ de 26 a 33 años, mientras que el mayor porcentaje, es decir, el $40 \%$ de encuestados corresponde a edades entre los 34 a 41 años, por otro lado, el $20 \%$ de encuestados tiene edades entre 42 a 49 años, finalmente, el $13 \%$ de encuestados tienen más de 50 años. 


\section{1. ¿Consume usted queso mozzarella?}

Tabla 5

¿Consume usted queso mozzarella?

\begin{tabular}{lll}
\hline Opciones & $\mathrm{Q}$ & $\%$ \\
\hline $\mathrm{Si}$ & 372 & $97 \%$ \\
\hline No & 11 & $3 \%$ \\
\hline Total personas & $\mathbf{3 8 3}$ & $\mathbf{1 0 0} \%$ \\
\hline
\end{tabular}

Nota: elaboración propia.

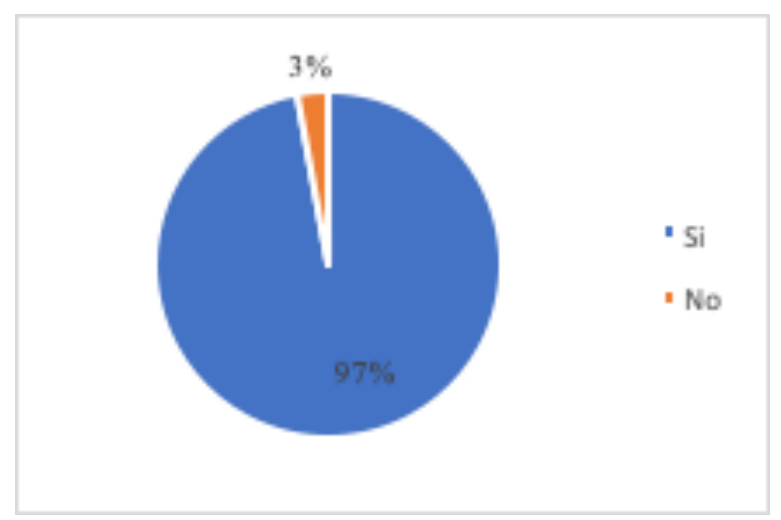

Figura 3. ¿Consume usted queso mozzarella?

\section{Interpretación:}

El $97 \%$ de los encuestados consumen queso mozzarella, mientras que el $3 \%$ no lo consume.

\section{2. ¿Cuántas libras de queso mozzarella consume mensualmente?}

Tabla 6

¿Cuántas libras de queso mozzarella consume mensualmente?

\begin{tabular}{lll}
\hline Opciones & Q & $\%$ \\
\hline 1 a 3 libras & 237 & $64 \%$ \\
\hline 4 a 6 libras & 102 & $27 \%$ \\
\hline 7 a 9 libras & 18 & $5 \%$ \\
\hline 10 o más libras & 15 & $4 \%$ \\
\hline Total personas & $\mathbf{3 7 2}$ & $\mathbf{1 0 0} \%$ \\
\hline
\end{tabular}

Nota: elaboración propia. 


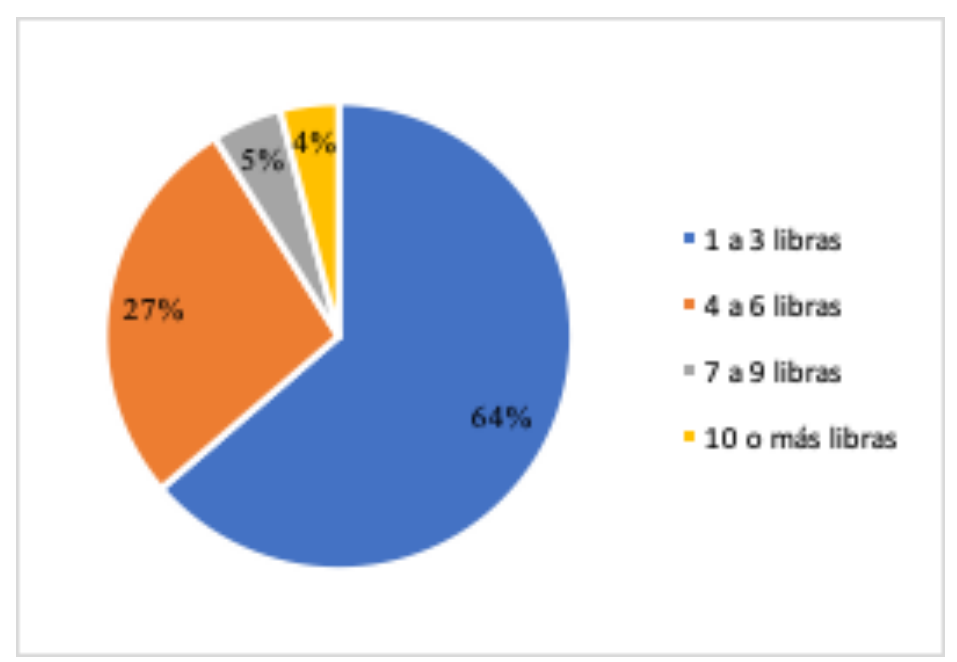

Figura 4. ¿Cuántas libras de queso mozzarella consume mensualmente?

\section{Interpretación:}

La mayoría de encuestados, es decir el $64 \%$ de ellos, consume de 1 a 3 libras de queso mozzarella al mes, el $27 \%$ de ellos consume 4 a 6 libras mensuales, el $5 \%$ de encuestados consume de 7 a 9 libras, mientras que el $4 \%$ consume 10 o más libras de queso mozzarella mensualmente.

3. ¿Con qué frecuencia compra queso mozzarella?

Tabla 7

¿Con qué frecuencia compra queso mozzarella?

\begin{tabular}{lll}
\hline Opciones & $\mathrm{Q}$ & $\%$ \\
\hline Diaria & 15 & $4 \%$ \\
\hline Semanal & 106 & $28 \%$ \\
\hline Quincenal & 102 & $27 \%$ \\
\hline Mensual & 149 & $40 \%$ \\
\hline Total personas & $\mathbf{3 7 2}$ & $\mathbf{1 0 0} \%$ \\
\hline
\end{tabular}

Nota: elaboración propia. 


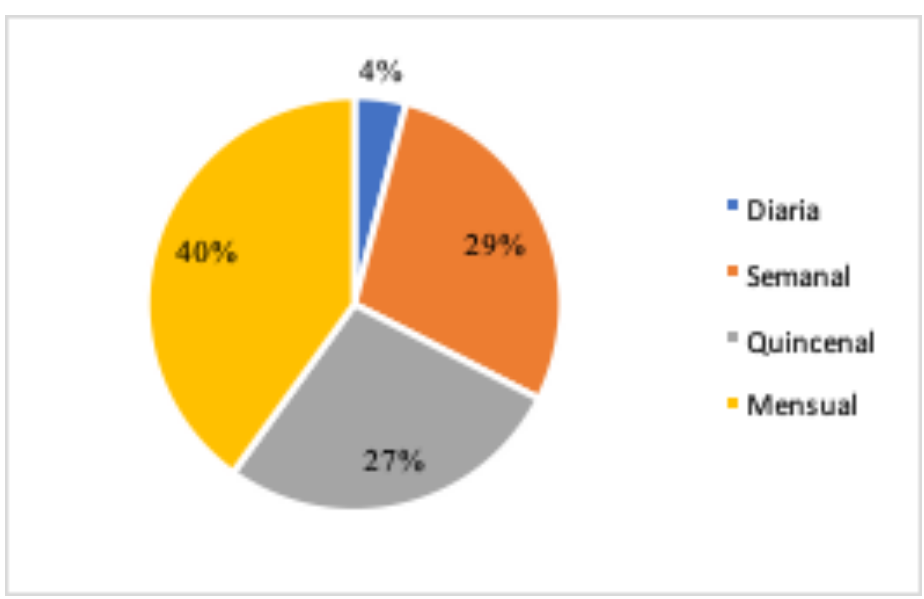

Figura 5. ¿Con qué frecuencia compra queso mozzarella?

\section{Interpretación:}

Tan solo el $4 \%$ de encuestados realizan compra diaria de queso mozzarella, el $28 \%$ lo adquiere semanalmente, el $27 \%$ de encuestados compran queso mozzarella quincenalmente, mientras que la mayoría de encuestados, es decir, el $40 \%$ de ellos realiza sus compras de queso mozzarella de manera mensual.

\section{4. ¿Cuál es el precio que cancela por una libra de queso mozzarella?}

\section{Tabla 8}

¿Cuál es el precio que cancela por una libra de queso mozzarella?

\begin{tabular}{|l|l|l|}
\hline Opciones & $\mathrm{Q}$ & $\%$ \\
\hline de 2 a 3 dólares & 84 & $23 \%$ \\
\hline de 3 a 4 dólares & 182 & $49 \%$ \\
\hline de 4 a 5 dólares & 73 & $20 \%$ \\
\hline más de 5 dólares & 33 & $9 \%$ \\
\hline Total personas & $\mathbf{3 7 2}$ & $100 \%$ \\
\hline
\end{tabular}

Nota: elaboración propia. 


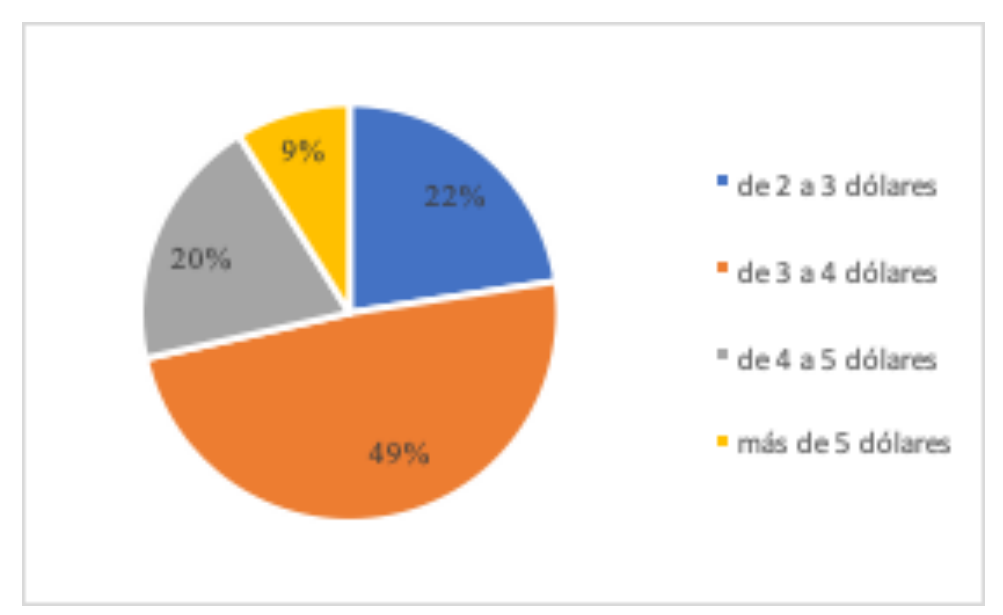

Figura 6. ¿Cuál es el precio que cancela por una libra de queso mozzarella?

\section{Interpretación:}

El 23\% de encuestados cancelan de 2 a 3 dólares por una libra de queso mozzarella, el $49 \%$ de encuestados pagan de 3 a 4 dólares, mientras que el $20 \%$ de encuestados cancelan de 4 a 5 dólares por una libra de queso, finalmente, tan solo el $9 \%$ de encuestados pagan más de 5 dólares por una libra de queso mozzarella.

\section{5. ¿Usualmente en dónde realiza su compra de queso mozzarella?}

Tabla 9

¿Usualmente en dónde realiza su compra de queso mozzarella?

\begin{tabular}{lll}
\hline Opciones & $\mathrm{Q}$ & $\%$ \\
\hline Tiendas de barrio & 26 & $7 \%$ \\
\hline Supermercados & 229 & $62 \%$ \\
\hline Distribuidores & 106 & $\mathbf{2 8 \%}$ \\
\hline Mercados populares & 4 & $1 \%$ \\
\hline Internet & 7 & $2 \%$ \\
\hline Total personas & $\mathbf{3 7 2}$ & $\mathbf{1 0 0} \%$ \\
\hline
\end{tabular}

Nota: elaboración propia. 


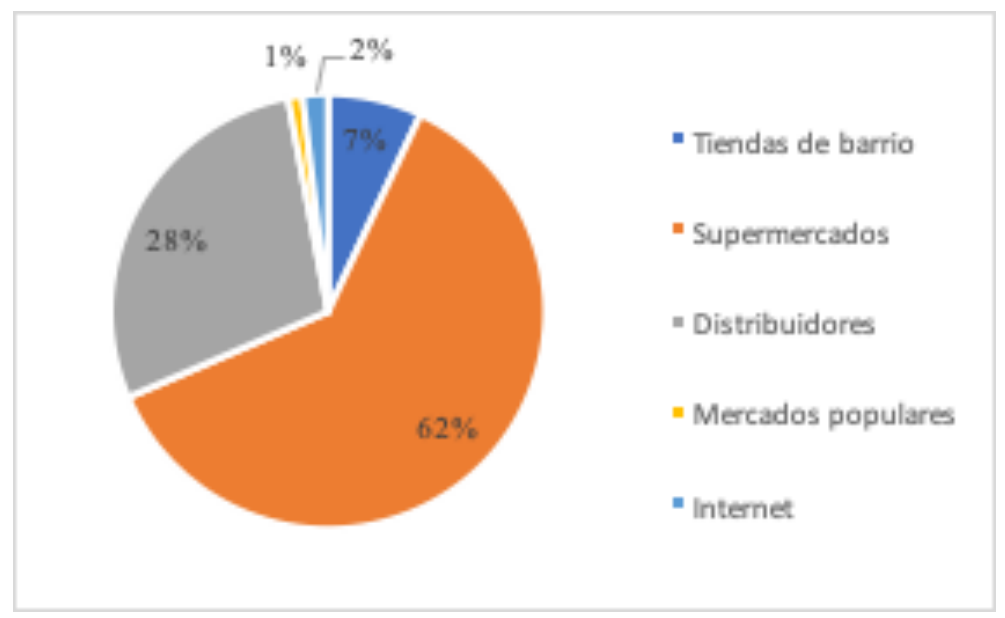

Figura 7. ¿Usualmente en dónde realiza su compra de queso mozzarella?

\section{Interpretación:}

El $62 \%$ de encuestados realizan sus copras de queso mozzarella en supermercados, y el $28 \%$ de ellos, lo realiza en distribuidoras, por otro lado, el $7 \%$ de encuestados realiza sus compras de queso en tiendas de barrio, finalmente el $1 \%$ y $2 \%$ de encuestados las realiza en mercados populares e internet, respectivamente.

\section{6. ¿Qué es lo que usualmente toma en cuenta antes de comprar queso?}

Tabla 10

¿Qué es lo que usualmente toma en cuenta antes de comprar queso?

\begin{tabular}{lll}
\hline Opciones & $\mathrm{Q}$ & $\%$ \\
\hline Precio & 51 & $14 \%$ \\
\hline Calidad & 251 & $68 \%$ \\
\hline Presentación & 15 & $4 \%$ \\
\hline Accesibilidad & 22 & $6 \%$ \\
\hline Marca & 33 & $9 \%$ \\
\hline Total personas & $\mathbf{3 7 2}$ & $\mathbf{1 0 0} \%$ \\
\hline
\end{tabular}

Nota: elaboración propia. 


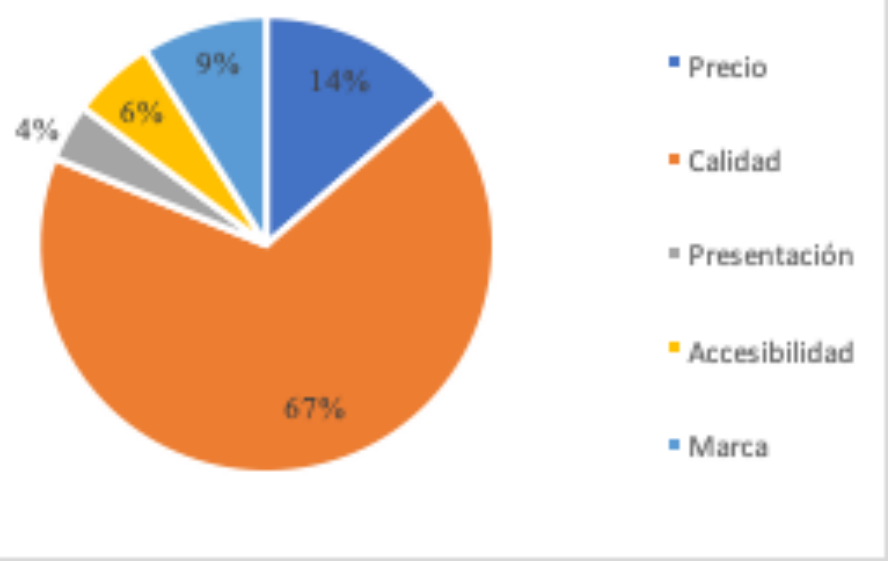

Figura 8. ¿Qué es lo que usualmente toma en cuenta antes de comprar queso?

\section{Interpretación:}

El $68 \%$ de encuestados indica que la calidad es lo más importante al momento de comprar queso mozzarella, el $14 \%$ considera el precio del producto, mientras que el $4 \%$, el $6 \%$ y el $9 \%$ de encuestados opinan que la presentación, accesibilidad y marca respectivamente, es lo usualmente toman en cuenta antes de realizar su compra de queso mozzarella.

\section{7. ¿Cuál de las siguientes marcas de queso es de su preferencia?}

Tabla 11

¿Cuál de las siguientes marcas de queso es de su preferencia?

\begin{tabular}{lll}
\hline Opciones & $\mathrm{Q}$ & $\%$ \\
\hline Alpina & 44 & $12 \%$ \\
\hline Kiosko & 131 & $35 \%$ \\
\hline Floralp & 26 & $7 \%$ \\
\hline Milma & 11 & $3 \%$ \\
\hline La Holandesa & 80 & $22 \%$ \\
\hline Salinerito & 58 & $16 \%$ \\
\hline Páramo & 4 & $1 \%$ \\
\hline Otras & 18 & $5 \%$ \\
\hline Total personas & 372 & $100 \%$ \\
\hline
\end{tabular}

Nota: elaboración propia. 


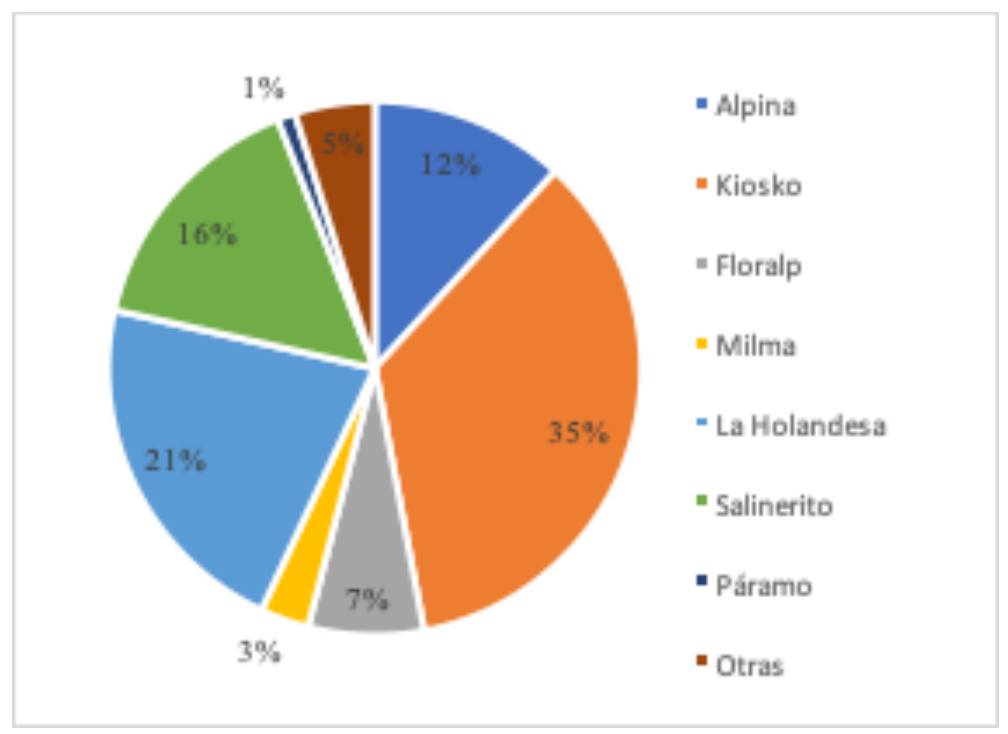

Figura 9. ¿Cuál de las siguientes marcas de queso es de su preferencia?

\section{Interpretación:}

El 12\% de encuestados prefieren la marca de queso Alpina, el 35\% prefieren Kiosko, el 7\% tienen preferencia por Floralp, el 3\% por Milma, el 21\% por la marca La Holandesa, el $16 \%$ de encuestados prefieren Salinerito, el 1\% de encuestados prefieren Páramo, mientras que el restante $5 \%$ prefiere otras marcas de queso.

\section{8. ¿Si se abriera un nuevo establecimiento dedicado a la distribución de queso mozzarella, el cual ofrezca servicio de entrega a domicilio, compraría en dicho lugar?}

Tabla 12

¿Si se abriera un nuevo establecimiento dedicado a la distribución de queso mozzarella, el cual ofrezca servicio de entrega a domicilio, compraría en dicho lugar?

\begin{tabular}{lll}
\hline Opciones & $\mathrm{Q}$ & $\%$ \\
\hline $\mathrm{Si}$ & 336 & $88 \%$ \\
\hline No & 47 & $12 \%$ \\
\hline Total personas & $\mathbf{3 8 3}$ & $\mathbf{1 0 0 \%}$ \\
\hline
\end{tabular}

Nota: elaboración propia. 


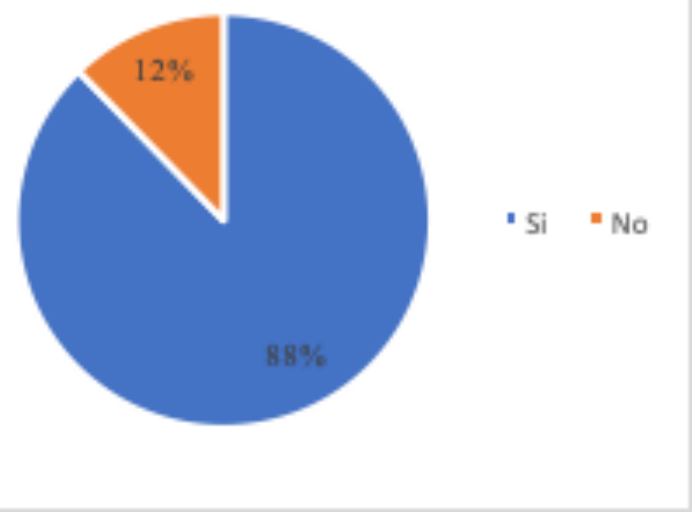

Figura 10. ¿Si se abriera un nuevo establecimiento dedicado a la distribución de queso mozzarella, el cual ofrezca servicio de entrega a domicilio, compraría en dicho lugar?

\section{Interpretación:}

El $88 \%$ de encuestados se encuentran interesados en adquirir queso mozzarella en un nuevo establecimiento de distribución que ofrezca servicios de entrega a domicilio, mientras que el restante $12 \%$ no estaría interesado.

\section{9. ¿Cuál variedad de queso mozzarella le gustaría consumir?}

Tabla 13

¿Cuál variedad de queso mozzarella le gustaría consumir?

\begin{tabular}{lll}
\hline Opciones & $\mathrm{Q}$ & $\%$ \\
\hline Bloque de mozzarella sabor natural & 189 & $50 \%$ \\
\hline Mozzarella laminado sabor natural & 102 & $27 \%$ \\
\hline Mozzarella con finas hierbas en bloque & 55 & $14 \%$ \\
\hline Mozzarella con finas hierbas laminado & 7 & $2 \%$ \\
\hline Mozzarella con ají en bloque & 11 & $3 \%$ \\
\hline Mozzarella con ají laminado & 15 & $4 \%$ \\
\hline Total personas & $\mathbf{3 7 9}$ & $\mathbf{1 0 0} \%$ \\
\hline
\end{tabular}

Nota: elaboración propia. 


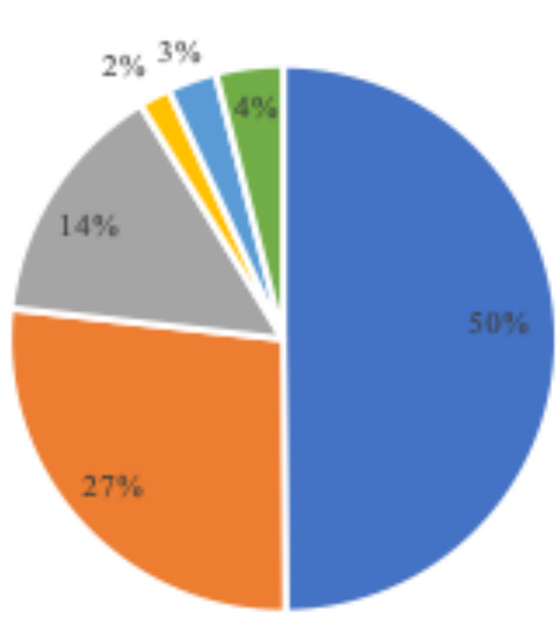

- Bloque de mozzarella sabor natural

- Mozzarella laminado sabor natural

- Mozzarella con finas hierbas en bloque

- Mozzarella con finas hierbas laminado

- Mozzarella con aji en bloque

- Mozzarella con ají laminado

Figura 11. ¿Cuál variedad de queso mozzarella le gustaría consumir?

\section{Interpretación:}

El 50\% de personas encuestadas están interesadas en consumir queso mozzarella en bloque sabor natural, mientras que el $27 \%$ de encuestados les gustaría consumir queso mozzarella laminado sabor natural, por otro lado, el 14\% de encuestados les gustaría consumir queso mozzarella en bloque con finas hiervas y tan solo el $2 \%$ de encuestados consumiría queso mozzarella laminado con finas hiervas, finalmente, el $3 \%$ y $4 \%$ de encuestados estarían interesados en consumir queso mozzarella con ají en bloque y laminado respectivamente

\section{0. ¿En qué presentación le gusta consumir queso mozzarella?}

Tabla 14 ¿En qué presentación le gusta consumir queso mozzarella?

\begin{tabular}{lll}
\hline Opciones & $\mathrm{Q}$ & $\%$ \\
\hline 250 gramos & 95 & $25 \%$ \\
\hline 500 gramos & 153 & $41 \%$ \\
\hline 750 gramos & 26 & $7 \%$ \\
\hline 1000 gramos & 40 & $11 \%$ \\
\hline más de 1000 gramos & 62 & $17 \%$ \\
\hline Total personas & 376 & $100 \%$ \\
\hline
\end{tabular}

Nota: elaboración propia. 


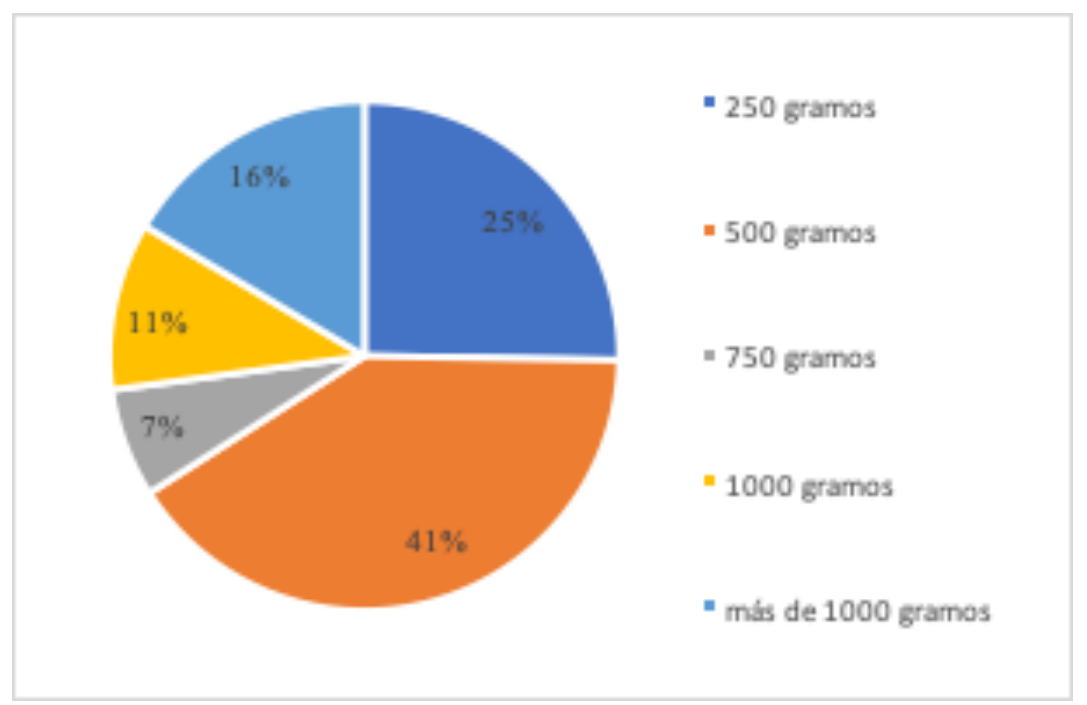

Figura 12. ¿En qué presentación le gusta consumir queso mozzarella?

\section{Interpretación:}

El 25\% de los encuestados indican su gusto por consumir queso mozzarella en presentación de 250 gramos, el $41 \%$ en cambio prefiere consumirlo en la presentación de 500 gramos, el $7 \%$ afirma preferir la presentación de 750 gramos, el 11\% de encuestados prefiere consumir queso mozzarella en presentaciones de 1000 gramos, finalmente al $16 \%$ de encuestados le gusta consumir queso mozzarella en presentaciones de más de 1000 gramos.

\section{CONCLUSIONES}

- En el mercado existen marcas reconocidas que elaboran y distribuyen queso mozzarella a nivel de todo el país, estas marcas cuentan con la preferencia de los consumidores, tal como lo refleja la pregunta No. 7 de la encuesta, mediante la cual se pudo conocer que Kiosco, La Holandesa Salinerito y Alpina son de las más reconocidas en el mercado y los consumidores usualmente las adquieren en supermercados y tiendas distribuidoras, lo que representa una desventaja para la compañía, debido a que los quesos que distribuirá no provienen de marcas reconocidas, sino de microempresas artesanales.

- La amenaza de entrada de nuevos competidores depende de las barreras que existan en el país para la creación de nuevos negocios con actividades iguales o similares al del presente proyecto, el nivel de inversión necesaria para la puesta en marcha del negocio, las políticas y reglamentos del Gobierno y del Municipio, los requerimientos del espacio físico, el nivel de dificultad para encontrar proveedores que permitan abaratar costos sin afectar la calidad de los productos, etc. 
- La entrada de productos y servicios sustitutos en el mercado conlleva a la reducción de precios para poder hacerle frente a la competencia y por lo tanto, menor rentabilidad.

- Actualmente, las marcas reconocidas de queso mozzarella manejan rangos de precio un poco más altos que aquellos con marcas artesanales que no son conocidas en el mercado, sin embargo, los precios siempre se mantienen dentro del rango que se manejará para la puesta en marcha del presente proyecto.

- Al constituirse el queso mozzarella como un producto alimenticio de consumo diario, llegando a ser altamente utilizado en negocios de comida rápida y al no existir muchas empresas que ofrezcan servicio de entrega a domicilio, se estima que el poder de negociación del cliente es una amenaza de nivel bajo.

- De acuerdo con la información obtenida mediante la aplicación de las encuestas, según la pregunta No. 5 , tan solo el $2 \%$ de personas adquieren queso mozzarella vía internet a empresas distribuidoras que ofrecen el mismo servicio, esto quiere decir que los servicios brindados por los supermercados, distribuidoras y tiendas de barrio ofrecen ese servicio sustituto que constituye una fuerte amenaza para el presente proyecto, ya que tienen la preferencia de los consumidores al momento de realizar la compra del producto, además, muchos de estos establecimientos comerciales brindan gran variedad de productos sustitutos al queso mozzarella, como lo son el queso fresco, queso chédar, queso con especias, queso vegano, etc.

- A los alrededores del Distrito Metropolitano de Quito existen muchas empresas dedicadas a la elaboración artesanal de queso mozzarella, cuyos productos finales tienen diferentes características y niveles de calidad de producto, además, casi todas las empresas proveedoras manejan rangos de precios parecidos, razón por la cual, el nivel de poder de negociación de los proveedores es bajo y no constituye una amenaza para la puesta en marcha de nuevos proyectos de negocios.

- Actualmente, el Gobierno Central se encuentra interesado en impulsar la apertura de nuevas fuentes de empleo, de emprendimientos y nuevos negocios que contribuyan al desarrollo económico del país, esto incluye políticas que faciliten la obtención de créditos para nuevos emprendedores, por lo cual, no solamente es beneficioso para el proyecto, también constituye una amenaza latente, debido a la facilidad que existe para la entrada de nuevos competidores al mercado.

- Las encuestas aplicadas en el mercado entregaron resultados favorables para la puesta en marcha de nuevos proyectos, ya que mediante sus resultados se pudieron conocer las características de consumo de los clientes, el tamaño de los empaques, el rango de precios más adecuado para el producto, el tipo de queso que los consumidores prefieren comprar y su frecuencia de consumo, toda esta información corresponde a un insumo importante que permitió lograr el cálculo de la oferta, demanda y demanda insatisfecha. 
- Es indispensable poner atención a las estrategias de marketing que se deben aplicar, para poder dar a conocer los productos y servicio de entrega a domicilio que ofrecen las empresas de queso mozzarella, de esta manera se podrá lograr el cumplimiento de las metas planteadas.

- Se recomienda realizar un estudio de mercado a partir del tercer año de la puesta en marcha de nuevos proyectos, que permitan conocer la factibilidad de expansión del negocio a otros sectores de la ciudad, con el fin de obtener un crecimiento del portafolio de clientes y en consecuencia, un crecimiento económico para la compañía.

- Para una correcta puesta en marcha de nuevos proyectos, es importante que el personal, conozca el direccionamiento estratégico de la compañía, a fin de que estos se identifiquen con los objetivos y metas de la empresa y de esta manera se pueden obtener los resultados esperados. 


\section{REFERENCIAS BIBLIOGRÁFICAS}

Acuerdo No. 01257, Reglamento de Prevención, Mitigación y Protección Contra Incendios del Ministerio de Inclusión Económica y Social. (23 de diciembre de 2008). Subsecretaría y Dlrección de Defernsa Contra Incendios. Quito, Ecuador: Registro Oficial Edición Especial 114,2-IV-2009.

Baca, U. (2013). “Preparación y Formulación de proyectos”. Bogotá: Ed. Norma.

Barreno, L. (2010). Manual de Formulación y Evaluación de Proyectos. Quito,.

Bustamante, E. (2009). La empresa. El Cid Editor.

Cabrera Figueroa, A., \& Palacios Villamizar, A. (2018). MODELO PLAN DE NEGOCIO PARA LA CREACIÓN DE UNA EMPRESA DE SERVICIOS DE AUTOMATIZACIÓN INTELIGENTE DE VIVIENDAS (DOMÓTICA), DE LOS ESTRATOS 4, 5 Y 6 DE LA LOCALIDAD DE SUBA DE LA CIUDAD DE BOGOTÁ. Bogotá: Universidad Externado de Colombia.

Cadena Lozano, J., \& Pozo Gaete, S. (2015). Producción y comercialización de queso amasado en la ciudad de Quito. Quito: UDLA.

Canseco Toasa, F. S. (2009). Plan de negocios para la creación de una empresa que preste servicios de: diseñeño, instalación, mantenimiento y asesoría en sistemas; eléctrico, electrónico de seguridad y telecomunicaciones, en el cantón rumiñahui. Escuela Politécnica Nacional.

Centro de la Industria Láctea del Ecuador. (2015). LA LECHE DEL ECUADOR-Historia de la lechería ecuatoriana. Quito: Centro de la Industria Láctea del Ecuador.

Centro de la Industria Láctea del Ecuador. (08 de abril de 2018). La producción de leche en el Ecuador. Obtenido de https://cilecuador.org/index.php/2018/04/08/produccionleche/

Chase, A. J. (2003). “Administración de la producción de Operaciones”, Pág. 119-130,. México,: Ed. Mc Graw Hill,.

Chiavenato, I. (1999). Administración de Recursos Humanos. Mc. Graw Hill.

CIL Ecuador. (10 de octubre de 2018). El Centro de la Industria Láctea del Ecuador respalda el uso de suero de leche. Obtenido de https://www.pichinchauniversal.com.ec/el-centro-de-la-industria-lactea-del-ecuadorrespalda-el-uso-de-suero-de-leche/

Conelec. (2013). Plan Maestro de Electrificación 2013 - 2022. Ministerio Coordinador de Sectores Estratégicos, Ministerio de Electricidad y Energía Renovable. 
Constante, J. (03 de agosto de 2016). El telégrafo. Obtenido de ¿Cómo se genera la energía eléctrica en Ecuador?: https://www.eltelegrafo.com.ec/noticias/punto/1/como-se-genera-la-energia-electrica-en-ecuador

Dirección Nacional de Estudios de Merecado. (2019). Informe del sector lácteo en Ecuador período 2013 - 2015. Superintendencia de Control del Poder de Mercado.

El Telégrafo. (22 de febrero de 2019). El consumo de lácteos en Ecuador aún es bajo. Obtenido de https://www. eltelegrafo.com.ec/noticias/economia/4/bajo-consumo-lacteos-ecuador

El Universo. (07 de octubre de 2018). Ecuador, líder regional en emprendimiento, por sexto año consecutivo. Obtenido de https://www.eluniverso.com/guayaquil/2018/10/07/nota/6986654/ecuador-lider-regional-emprendimiento-sexto-ano-consecutivo

Espinoza Montes, F. (2015). La tesis universitaria. Huancayo-Perú: Master SAC.

Fayol, H. (1987). Administración Industrial y General. Buenos Aires: El Ateneo.

Federico. (s.f.). Estudio Financiero. Obtenido de Zona Económica: https://www.zonaeconomica.com/estudio-financiero

Ferrell, O., Hirt, F., \& Ferrell, L. (2010). Introducción a los negocios en un mundo cambiante. México D.F.: Mc Graw Hill.

Fischer, L., \& Espejo, J. (2011). Mercadotecnia. México: Mc. Graw Hill.

Flores, F. (27 de febrero de 2019). Los retos de emprender en Ecuador y cómo superarlos. Obtenido de Edes Business school UTPL: https://edes.utpl.edu.ec/blog-post/retos-emprender-ecuador/

Flores, J. (s.f.). MICROEMPRESA. Obtenido de Derecho Ecuador: https://www.derechoecuador.com/microempresa

Fondo Moneratio Internacional. (21 de marzo de 2019). Análisis del nuevo plan económico de Ecuador. Obtenido de https://www.imf.org/es/News/Articles/2019/03/20/NA032119-Ecuador-New-Economic-Plan-Explained

Gestión Digital. (06 de diciembre de 2018). Economía y Finanzas. Obtenido de https://revistagestion.ec/economia-y-finanzas-analisis/quito-concentra-su-economia-en-los-servicios

González, N., López, E., Aceves, J., Celaya, R., \& Nancy, A. (s.f.). Que integra el estudio financiero en un plan de Negocios . Obtenido de Itson.mx: https://www.itson.mx/publicaciones/pacioli/Documents/no56/estudio_financiero.pdf

Hernández León, R., \& Coello Gozález, S. (2006). El proceso de investigación científica. La Habana: Editorial Universitaria Cubana. 
Hernández, R., Fernández , C., \& Baptista, P. (2015). Metodología de la investigación. México D.F.: McGraw Hill. Instituto Nacional de Estadítica y Censos. (2014). Resumen de la Comisión Especial de Estadística de Seguridad, Justicia, Crimen y Transparencia. Quito: INEC.

Kothler, F. (2006). Estrategias de Marketing. México: Ed. Mc Graw Hill.

Kotler, P., \& Keller, K. (2006). Dirección de Marketing. México: PEARSON.

Kotler, P., Armstrong, G., \& Cruz, I. (2006). Marketing. México: Pearson-Prentice Hall4.

Lácteos LATAM. (11 de agosto de 2017). Evaluación higiénico - sanitaria de la quesera artesanal COD.Q6. Obtenido de https://www.lacteoslatam.com/paises/80-ecuador/3675-evaluaci\%C3\%B3n-higi\%C3\%A9nico-sanitaria-de-la-quesera-artesanal-cod-q6.html

Lamb, C., Hair, J., \& McDaniel, C. (2002). Marketing. Mc. Graw Hill.

Longenecker, J., Palich, L., Petty, W., \& Hoy, F. (2012). Administración de pequeñas empresas. México D.F.: CENGAGE Learning.

López, R., Barberán, F., \& Briones, J. (2015). Plan de negocios de servicios eléctricos "Construcciones Totales””'. Guayaquil: Universidad Politécnica Salesiana.

Más Leche - CIL. (17 de julio de 2017). Ganadería Sostenible en Ecuador. Obtenido de http://webcache.googleusercontent.com/search?q=cache:-u7q9o3UKX0J:masleche.ec/ganaderia-sostenible-en-ecuador/+\&c$\mathrm{d}=1 \& \mathrm{hl}=\mathrm{es}-419 \& \mathrm{ct}=\mathrm{clnk} \& \mathrm{gl}=\mathrm{ec}$

Materiales de Lengua. (s.f.). ¿Qué es una entrevista? Obtenido de Sites.google.com: https://sites.google.com/ site/entrevistaconelexperto/-que-es-una-entrevista

Mejía, E. J. (2016). Aplicación del Modelo Jessi ampliado con muestreo de áreas para la estimación de demanda potencial para el segmento de Millenials en Ecuador. Revista Publicando, 3(7), 333-345. .

Ministerio de Comercio Exterior e Inversiones. (27 de julio de 2017). 95\% de empresas en Ecuador son MiPymes. Obtenido de Ministerio de Comercio Exterior e Inversiones: https://www.comercioexterior.gob.ec/95de-empresas-en-ecuador-son-mypimes/

Morán Macías, L. (2014). PLAN DE NEGOCIOS PARA LA CREACIÓN DE UNA EMPRESA PRODUCTORA Y COMERCIALIZADORA DE QUESO FRESCO Y MADURO EN EL CANTÓN LA MANÁ. La Maná: Universidad Técnica de Cotopaxi.

Moreno Cáceres, L. (2016). Plan de negocios para la creación de una empresa de producción y comercialización de queso mozzarella del sector sur de la ciudad de Quito. Quito: Universidad Tecnológica "Indoamérica”. 
OKDIARIO. (20 de agosto de 2017). Beneficios y propiedades nutricionales de la Mozzarella. Obtenido de https://okdiario.com/salud/mozzarella-propiedades-63894

OkDiario. (25 de julio de 2018). ¿Qué es la oferta y la demanda? Obtenido de https://okdiario.com/curiosidades/ que-oferta-demanda-2758201

Organización de las Naciones Unidas para la Alimentación y la Agricultura. (s.f.). Portal lácteo. Obtenido de http://www.fao.org/dairy-production-products/products/tipos-y-caracteristicas/es/

Orozco, M. (16 de febrero de 2015). Un tercio de la producción láctea se dedica al queso. Obtenido de https:// www.revistalideres.ec/lideres/ecuador-produccion-lactea-queso.html

Pilco Mosquera, W., \& Ruiz Mancero, L. (2015). La invertigación de mercados com ouna disciplina estratégica. Riobamba: ESPOCH.

ProEcuador. (14 de mayo de 2018). TICS. Obtenido de https://www.proecuador.gob.ec/tics/

QuestionPro. (s.f.). ¿Qué es una encuesta? Obtenido de QuestionPro: https://www.questionpro.com/es/encuesta.html

Ramórez López, C., \& Vélez Ruiz , J. (2012). Quesos frescos: propiedades, métodos de determinación y factores que afectan su calidad. Puebla, México: Departamento de Ingeniería Química, Alimentos y Ambiental, Universidad de las Américas Puebla.

Registro Oficial N¹4. (14 de junio de 2017). Registro Oficial. Quito, Ecuador: Corte Constitucional.

Reglamento de Seguridad del Trabajo Contra Riesgos en Instalaciones de Energía Eléctrica. Acuerdo No. 013. (22 de enero de 1998). Registro Oficial. Quito, Ecuador: Comité Interinstitucional de Seguridad e Higiene del Trabajo.

Relación empresa-clientes. (s.f.). El cliente.

Rodríguez, G., Gil, J., \& García, E. (1996). Metodología de la Investigación Cualitativa. Granada: Aljibe.

Romero Hidalgo, O., Hidalgo Sánchez, A., \& Correa Guaicha, H. (2018). Plan de Negocios. Un enfoque práctico en el sector comercio. Machala: Editorial UTMACH.

Rosero Márquez, F., \& Rodríguez Bermúdez, E. (2012). ESTUDIO DE VIABILIDAD TÉCNICA Y DE MERCADO PARA UNA EMPRESA DE SERVICIOS ELÉCTRICOS. Pereira: Universidad Tecnológica de Pereira Facultad de Tecnología.

Sapag, C. (1995). Preparación y Evaluación de Proyectos. Bogotá: McGraw Hill. 
Sapag, C. N. (2007). “Formulación y Evaluación de Proyectos”, Pág. 15. Madrid: Ed. Prentice Hall.

Servicio Ecuatoriano de Normalización. (2017). Prologo. Construimos Calidad, 3.

Sobrero, F. (2009). Análisis de Viabilidad: La cenicienta en los Proyectos de Inversión. FCE-UNL.

Solo quesos. (s.f.). Queso Mozzarella. Obtenido de http://soloqueso.com/queso-mozzarella/

Tapia, D. (15 de junio de 2017). ECUADOR, PAÍS LIDER EN EMPRENDIMIENTOS EN AMÉRICA LATINA. Obtenido de https://workingup.com.ec/2017/06/15/ecuador-pais-lider-emprendimientos-america-latina/

Tapia, E. (9 de octubre de 2018). Mipymes generan el 20\% de los ingresos del tejido empresarial. Obtenido de Líderes: https://www.revistalideres.ec/lideres/mipymes-ingresos-superintendencia-companias-informe. html

Ulcuango Conlago, M. (2017). MODELO DE GESTIÓN PARA EL ÁREA DE PRODUCCIÓN DE LA MICROEMPRESA DE LÁCTEOS PRODULUTSA UBICADA EN LA CIUDAD DE CAYAMBE. Quito: Universidad Central del Ecuador.

Unidad de Estadísticas Agropecuarias. (2017). Encuesta de Superficie y Proeuccion Agropecuaria Continua. INEC.

Valera, R. (27 de septiembre de 2010). Blogger. Obtenido de ESTUDIO DE FACTIBILIDAD Y PROYECTOS: http://estudiodefactibilidadyproyectos.blogspot.com/2010/09/factibilidad-y-viabilidad.html

Weinberger, K. (2009). Plan de negocios, herramienta para evaluar la viabilidad de un negocio. Perú: Ministerio de la Producción.

WordPress. (18 de diciembre de 2013). ¿Qué Es El Marketing Social? Obtenido de https://marketingparados. wordpress.com/tag/demanda-insatisfecha/ 